Revue d'histoire du XIXe siècle

Société d'histoire de la révolution de 1848 et des

révolutions du XIXe siècle

Religion, politique et culture au XIXe siècle

\title{
La politique religieuse de la Seconde République
}

Jacqueline Lalouette

URL : http://journals.openedition.org/rh19/619

DOI : $10.4000 /$ rh19.619

ISSN : $1777-5329$

Éditeur

La Société de 1848

Édition imprimée

Date de publication : 1 juin 2004

Pagination : 79-94

ISSN : 1265-1354

Référence électronique

Jacqueline Lalouette, "La politique religieuse de la Seconde République », Revue d'histoire du XIXe siècle [En ligne], 28 | 2004, mis en ligne le 07 avril 2008, consulté le 19 avril 2019. URL : http:// journals.openedition.org/rh19/619; DOI : 10.4000/rh19.619

Ce document a été généré automatiquement le 19 avril 2019

Tous droits réservés 


\title{
La politique religieuse de la Seconde République
}

\author{
Jacqueline Lalouette
}

1 Quelques épisodes particulièrement célèbres - comme le transfert des calices, des ciboires et du Christ de la chapelle des Tuileries à l'église Saint-Roch par les insurgés euxmêmes, durant la journée du 24 février ${ }^{1}-$ illustrent le caractère religieux de la Révolution de 1848, qui «se fit contre le trône, mais respecta l'autel ${ }^{2}$. Les représentations que les «quarante-huitards » avaient du Christ, qu'ils voyaient comme un «sans-culotte », comme le premier des républicains ou des socialistes, témoignent, elles aussi, des rapports qui s'établirent à cette époque entre religion et politique ${ }^{3}$. De son côté, l'Église accueillit favorablement les événements de Février. Le 7 mars, Mgr Affre assura Dupont de l'Eure «du loyal concours du clergé», ce à quoi le chef du Gouvernement provisoire répondit par ces mots rassurants : «La liberté et la religion sont deux sœurs également intéressées à bien vivre ensemble ${ }^{4}$; enfin, le clergé catholique donna bien volontiers sa bénédiction aux arbres de la Liberté ${ }^{5}$.

2 Par ailleurs, en diverses circonstances, la Seconde République associa l'Église à ses actes ou à ses fastes. Ainsi, dès le 29 février 1848, le Gouvernement provisoire engagea « M. l'archevêque de Paris et tous les évêques de la République à substituer à l'ancienne formule de prières les mots Domine, salvam fac Rempublicam " ${ }^{6}$; au mois de novembre, le ministre des Cultes pria les archevêques et les évêques de faire célébrer dans toutes les paroisses de leur diocèse un Te Deum «pour appeler les bénédictions du ciel sur cette Constitution, qui fonde et proclame la République démocratique $»^{7}$.

Cependant, les nouveaux textes ou projets relatifs au statut des cultes et aux rapports devant exister entre ceux-ci et l'État sont généralement passés sous silence par les historiens de 1848, dont l'attention s'est plus volontiers portée sur des questions d'ordre politique et social que sur des questions d'ordre religieux ${ }^{8}$. Pourtant, la Révolution de 1848 amena très rapidement quelques modifications majeures dans la situation des fidèles pratiquant un culte minoritaire ; puis, en supprimant toute référence au catholicisme, la Constitution du 4 novembre introduisit une rupture, temporaire certes, mais 
fondamentale dans l'histoire religieuse institutionnelle de la France ; enfin, la révision du Concordat de 1802 fut entamée, mais n'aboutit pas.

4 Lorsqu'éclata la Révolution de 1848, depuis déjà de longues années la séparation de l'Église et de l'État était réclamée par des voix diverses. Outre l'Avenir, les catholiques libéraux, que l'encyclique Mirari vos avait ensuite amenés à abandonner cette revendication, disposaient du journal La Tribune catholique; depuis 1831 des protestants ou des personnalités proches du protestantisme menaient une campagne séparatiste dans le journal Le Semeur ${ }^{9}$. Il faudrait citer des organes spécialisés dans les questions d'ordre religieux, comme le Journal de la liberté religieuse, et des journaux généralistes, comme Le Siècle, mentionner divers ouvrages, comme ceux d'Alexandre Vinet ${ }^{10}$, dont l'influence fut considérable, et bien d'autres écrits encore. Parfois, à la Chambre des députés, une personnalité en vue s'aventurait à réclamer soit la suppression du budget des cultes, considérée comme un préliminaire de la séparation - comme Audry de Puiraveau en 1832 et $1833^{11}$-, soit la séparation elle-même, comme Isambert ${ }^{12}$ ou Lamartine en $1845^{13}$ . De son côté, en 1836, Charles Teste avait publié à Bruxelles un Projet de constitution républicaine qui reprenait les termes utilisés par les Conventionnels en 1795, selon lesquels « la République ne salarie aucun culte " ${ }^{14}$, formule promise à un bel avenir grâce au législateur de 1905. Les démocrates considéraient alors la liberté de conscience comme « la première et la plus inviolable ${ }^{15}$ des libertés et voyaient dans la liberté des cultes sa conséquence logique et indispensable. Ces deux libertés figuraient au premier plan des motifs invoqués pour transformer le statut des cultes; tel qu'il était fixé par le Concordat et par la Charte de 1830, celui-ci introduisait bien des inégalités entre Français catholiques et Français se réclamant d'autres cultes, reconnus ou non reconnus.

En effet, en dépit de l'article 5 de la charte de 1830, qui garantissait la liberté des cultes, et de l'article 6, qui avait ramené la religion catholique du statut de religion de l'État - que lui avait conféré la Restauration ${ }^{16}$-, à celui de religion de « la majorité des Français » ${ }^{17}$, tous les Français n'étaient pas libres de pratiquer leur religion comme ils l'entendaient dans la France de Louis-Philippe, et les magistrats n'hésitaient pas à poursuivre des ministres de cultes non reconnus, et parfois même de cultes reconnus, en invoquant les articles 290, 291 et 294 du Code pénal et la loi du 10 avril 1834. Quelques affaires méritent d'être rapportées. En 1833, l'Église catholique française de Clichy avait été fermée sur ordre du sous-préfet, ce qui avait amené les habitants à entamer une lutte et à construire des barricades ${ }^{18}$. En 1836, M. Pillot, ministre d'une Église française unitaire et réformatrice installée au Pecq fut à son tour poursuivi et condamné à six mois de prison ${ }^{19}$ . En 1837, les habitants de Senneville (Seine-et-Oise), en guerre ouverte avec le desservant de leur paroisse, avaient fait appel à M. Laverdet, ministre d'une église schismatique, l'Église française, et avaient bâti une chapelle pour célébrer leurs cérémonies. Les autorités en avaient ordonné la fermeture et avaient poursuivi M. Laverdet, inculpé d'usurpation de costume d'un ministre de l'Église romaine et de constitution d'une association de plus de vingt personnes; condamné en première instance par le tribunal de Mantes, en appel par celui de Versailles, ce prêtre schismatique s'était pourvu en cassation, mais son pourvoi avait été rejeté le 22 juillet 1837. Entêtés, les habitants de Senneville s'étaient convertis au protestantisme et avaient fait venir M. Roussel, un ancien pasteur de Genève, qu'un détachement de gendarmerie obligea à quitter les lieux et qui fut poursuivi et condamné en 1842 ; des pasteurs de l'Église de la Confession d'Augsbourg et des pasteurs de l'Église réformée protestèrent alors solennellement auprès $\mathrm{du}$ Garde des Sceaux ${ }^{20}$. La même mésaventure survint au pasteur de l'Église 
réformée de Dijon, qui, en 1846, avait célébré le culte dans une commune voisine, Ahuy, en un lieu qui n'était pas établi officiellement ${ }^{21}$. En 1847 , ce fut un pasteur baptiste établi à Chauny (Oise) et deux fidèles de ce culte qui apprirent à leurs dépens que l'article 5 de la Charte ne protégeait pas véritablement les adeptes des cultes minoritaires; le 22 janvier, le tribunal de Laon les condamna chacun à 300 francs d'amende, peine qui fut confirmée en appel par la Cour royale d'Amiens, le 25 mars ${ }^{22}$. De tels exemples pourraient être multipliés; il ne faut, par ailleurs, pas omettre le cas de tous les individus condamnés dans des affaires de procession, en général pour ne s'être pas découverts au passage du Saint-Sacrement.

6 Dans l'état actuel de nos recherches, il nous est impossible de préciser combien de personnes étaient emprisonnées pour un délit à caractère religieux en 1848 et combien se trouvaient sous le coup d'une amende dont ils devaient s'acquitter. Elles n'étaient probablement pas très nombreuses, mais il faut croire que les condamnations prononcées pour ce type d'infraction avaient puissamment ému l'opinion républicaine puisque l'un des premiers actes du Gouvernement provisoire se rapporta à la liberté des cultes. Le 10 mars 1848, fut publié le décret suivant : «Le gouvernement provisoire, convaincu que de toutes les libertés, la liberté de conscience est la plus précieuse et la plus sainte, arrête : «Les citoyens détenus par suite de condamnations prononcées contre eux pour faits relatifs au libre exercice du culte, seront immédiatement rendus à la liberté, s'ils ne sont retenus pour une autre cause. Toute poursuite commencée est abolie. Remise est faite des amendes prononcées, et non encore acquittées. Le ministre de la Justice et le ministre des finances sont chargés de l'exécution du présent décret $"{ }^{23}$.

7 La Constitution du 4 novembre 1848 offre trois passages significatifs en matière de religion. Le préambule s'ouvre par la formule «En présence de Dieu et au nom du peuple français, l'Assemblée nationale proclame... » ${ }^{24}$. Lorsque le préambule fut discuté, dans la séance du 29 août 1848, plusieurs des amendements proposés portaient sur les mots « en présence de Dieu ». Mgr Fayet, évêque d'Orléans ${ }^{25}$, proposa la version « Au nom de Dieu », qui, si elle avait été acceptée, aurait modifié la conception de la souveraineté. JosephLouis Target souhaitait ajouter après le mot de «Dieu " la précision « et au nom de la liberté qu'il donne à tous ». À l'expression " en présence », le citoyen Roujat ${ }^{26}$ préférait celle de "sous la protection" et Jean Reynaud ${ }^{27}$ celle de «sous l'invocation». Enfin, Victor Guichard avait rédigé un nouveau préambule qui commençait ainsi : «En présence de Dieu, principe de toute justice, et au nom du peuple français ${ }^{28}$. L'article VIII du préambule établit que "la République doit protéger le citoyen dans [...] sa religion"; aucun des amendements proposés pour modifier cet article ne porta sur la protection que la République devait apporter aux citoyens en matière religieuse, la question du travail et de l'assistance monopolisant l'intérêt.

8 L'article 7 du corps de la Constitution stipule que « chacun professe librement sa religion, et reçoit de l'État, pour l'exercice de son culte, une égale protection ». Il ajoute, dans son second alinéa, que « les ministres, soit des cultes actuellement reconnus par la loi, soit de ceux qui seraient reconnus à l'avenir, ont le droit de recevoir un traitement de l'État ». Cet article introduit deux innovations majeures par rapport aux deux textes constitutionnels précédents. Tout d'abord, "la religion catholique, apostolique et romaine » n'est plus nommée, ni comme religion d'État, ni comme religion de la majorité des Français ; elle est incluse dans l'ensemble des religions protégées - c'est-à-dire toutes les religions - et dans le groupe plus réduit des religions reconnues. Ensuite, le législateur a décidé que le nombre de ces dernières n'était pas invariable et, qu'à l'avenir, 
d'autres cultes pourraient accéder à ce statut. Cette rédaction définitive de l'article 7 diffère de celle qu'avait proposée le comité de la Constitution; dans sa version primitive, le second alinéa réservait le versement d'un traitement par l'État aux seuls «ministres des cultes reconnus par la loi » et n'envisageait pas d'accorder cette qualité à de nouveaux cultes. Lors de la discussion, deux amendements visèrent non à un accroissement possible des cultes concernés, mais au contraire à la suppression du traitement pour tous les cultes. Pierre Leroux désirait que l'article 7 fût ramené à cette simple phrase: "Chacun professe librement sa religion " ${ }^{29}$. Jean Lavallée, quant à lui, s'orientait vers une rédaction rappelant celle de l'article 354 de la constitution de 1795 avec un amendement ainsi rédigé : «Nul citoyen ne peut être forcé de contribuer aux frais d'un culte ; la République n'en salarie aucun ${ }^{30}$. Isambert proposa un amendement - qu'il retira avant le vote - qui rendait nécessaire le vote d'une loi pour régler « les subventions de l'État en faveur des églises établies ainsi que les rapports de ces églises avec l'État ". L'idée contraire visant à subventionner d'autres cultes dans l'avenir ${ }^{31}$ fut introduite par Étienne-Ursin Bouzique ${ }^{32}$, qui retira lui aussi son amendement, et par Pierre-Siméon Bourzat - dont Victor Hugo a parlé dans Histoire d'un crime- qui appartenait «à la gauche la plus avancée » ${ }^{33}$. Son amendement comptait quatre alinéas. Les deux premiers établissaient la liberté des cultes, qui étaient tous assurés de bénéficier de la protection de l'État, mais qui devaient se soumettre aux lois et aux règlements "nécessaires au maintien de l'ordre et de la paix publique ». Le troisième exprimait une vive hostilité envers les congrégations religieuses, qui ne pouvaient «s'établir que dans les formes et sous les conditions déterminées par une loi spéciale d'autorisation », ce qui aurait provoqué la dissolution de toutes les congrégations tolérées, mais non autorisées. Enfin, d'après le quatrième amendement, « les ministres des cultes qui seront reconnus à l'avenir ne pourront recevoir de traitement qu'en vertu d'une loi de révision de la Constitution ». Finalement, ce fut Vivien ${ }^{34}$ qui, au nom du comité de la Constitution, proposa la nouvelle rédaction de l'article 7, qui fut adoptée pour le texte définitif.

9 Mais les principales modifications dans le domaine religieux faillirent être apportées par la révision du Concordat conclu avec l'Église catholique en 1802. Certes, comme le rappela Isambert, l'Assemblée constituante ne voulut pas «placer au rang de ses lois organiques la révision du Concordat et des articles qui l'ont suivi » ${ }^{35}$, mais en son sein se forma un comité des Cultes chargé d'étudier certaines questions ${ }^{36}$. Présidé tout d'abord par Vivien, puis par Mgr Parisis, évêque de Langres, ce comité comptait quarante-deux membres ${ }^{37}$, parmi lesquels figuraient six ecclésiastiques : trois évêques - Mgr Parisis, déjà nommé, Mgr Graveran, évêque de Quimper et Mgr Fayet, évêque d'Orléans ${ }^{38}$ - et trois abbés, les abbés Fournier, Fréchin, Moutou ${ }^{39}$. Parmi les laïcs, certains étaient connus pour leur appartenance au catholicisme libéral - comme Frédéric Arnaud de l'Ariège ${ }^{40}$, qui en était le vice-président -, d'autres pour leur catholicisme intransigeant, comme MM. Bouhier de l'Écluse ou de Charancey, d'autres enfin pour leur anticléricalisme, comme Isambert. Le protestantisme était représenté par un seul membre, M. Mispoulet ${ }^{41}$. Tout ce qui a trait à l'existence et aux travaux du comité ne nous est malheureusement connu que par le compte-rendu qu'en fit Pierre Pradié ${ }^{42}$, qui entendit écrire en "historien fidèle et impartial ${ }^{43}$, mais négligea de donner quelques informations élémentaires. Dans l'état actuel de notre documentation, nous ne connaissons pas la date à laquelle cessèrent les travaux de ce comité des Cultes et les raisons pour lesquelles ils ne furent pas pris en compte. La dernière réunion mentionnée par Pierre Pradié date du 26 mars 1849 ; elle précède donc de quelques semaines les élections législatives et la séparation de l'Assemblée constituante. 
$10 \mathrm{Au}$ comité des Cultes, certaines voix se firent entendre en faveur de la séparation ${ }^{44}$, mais la majorité de ses membres croyaient à la nécessité et à la possibilité d'une entente entre Rome et la République et souhaitaient voir s'ouvrir des négociations entre Paris et le Saint-Siège, afin de donner de nouvelles bases, plus libérales, au Concordat, notamment en modifiant les articles organiques, voire en les abrogeant "pour faire place à un nouveau concordat ${ }^{45}$. Ils repoussaient aussi bien l'idée d'une séparation complète entre l'Église et l'État que celle d'une subordination de l'Église à l'État pour adopter la formule d'« une indépendance réciproque du pouvoir spirituel et du pouvoir temporel, chacun dans leur sphère, tout en conservant entre ces deux pouvoirs les liens les plus intimes ", grâce à un concordat «librement consenti » par les deux partenaires ${ }^{46}$. La République étant « le gouvernement de tous, par tous et pour tous », ils ne concevaient pas l'idée d'en exclure quiconque ; l'Église, comme «les partis les plus extrêmes", devait y trouver sa place ${ }^{47}$. Cette conviction était d'autant plus forte que le clergé se montrant bienveillant, " aucun républicain sensé et intelligent ne pouvait conserver les injustes méfiances de l'ancienne opposition contre la religion»; tout républicain devait comprendre "que la religion est le premier intérêt du peuple et le fondement le plus solide de la République » ${ }^{48}$. Le comité espérait que ses travaux ouvriraient « la période de l'accord, de l'alliance, de la fraternité entre les cultes et l'État ${ }^{49}$. Pour Pierre Pradié, le comité avait inauguré « un droit nouveau dans les rapports de l'Église avec la puissance séculière » et mis en lumière " des principes que les querelles du Sacerdoce et de l'Empire avaient obscurcis » ${ }^{50}$.

11 Ces principes étant posés, la majorité du comité présentait la religion comme « une base indispensable de toute société régulière » et considérait que le culte, " mis au rang des nécessités sociales " ${ }^{51}$, devait bénéficier d'un budget lui permettant de subvenir à ses besoins matériels. Ses membres estimaient que le peuple, "habitué à considérer l'entretien matériel des cultes comme une charge de l'État », verrait d'un mauvais œil la suppression du budget des cultes et qu'une telle mesure ne serait pas « propre à créer des amis à la République et aux institutions qu'elle est appelée à fonder ${ }^{52}$.

12 Après lecture d'un rapport du représentant Chapot, présenté dans la séance du 15 juin 1848, le comité se prononça unanimement pour le maintien du budget des cultes, tout en lui apportant certaines modifications. La proposition fut avancée de réduire le traitement des cardinaux et des archevêques, de ramener celui de l'archevêque de Paris de 40000 à 25000 francs, traitement perçu par les maréchaux de France et par le premier président de la Cour de cassation; mais, firent valoir certains représentants, les frais de représentation de l'archevêque ainsi que les sommes qu'il dépensait en actes de bienfaisance ou en frais de fondations pieuses représentaient un total considérable dont on ne pouvait pas ne pas tenir compte. Les bourses pour les séminaristes, les pensions, allocations et secours pour les ecclésiastiques âgés ou infirmes, pour d'anciennes religieuses, pour des prêtres n'ayant "repris de service actif depuis 1802 » furent globalement maintenus, de même que les sommes affectées à l'entretien des édifices diocésains ; en revanche les secours versés à divers établissements ecclésiastiques furent ramenés de 156300 à 100000 francs. Le comité ne se montra pas généreux envers les cultes protestants et le culte israélite. Dans la préparation du budget de 1848, les sommes allouées aux pasteurs avaient été réduites de 12000 francs. Un membre du Comité « argu [a] de la dissémination des protestants et des frais de déplacement " que cet état de choses entraînait pour les pasteurs pour demander le rétablissement de la somme antérieure ; sans s'opposer franchement à une telle demande, le comité se prononça pour un ajournement de sa décision. Quant aux israélites, ils n'obtinrent pas non plus 
satisfaction. Une ordonnance du 19 octobre 1847 avait porté le traitement des rabbins de 300 à 500 francs et le Consistoire central demanda qu'il passât à 1000 francs; cette demande, qui parut «subite » et " exorbitante », fut repoussée. De même, le traitement de 4000 francs demandé pour le grand rabbin de Paris ne fut pas accordé, pour défaut de procédure administrative.

Diverses questions relatives à l'organisation interne de l'Église, au cursus et à la carrière des ecclésiastiques furent longuement soupesées, à commencer par le mode de nomination des évêques ${ }^{53}$. Quelques membres estimaient qu'il appartenait à l'État seul de statuer en la matière, "qu'il ne pouvait y avoir deux États dans l'État ", que ce dernier n'avait pas à traiter avec l'Église comme avec un État souverain; pour eux, bénéficiant d'un traitement versé par l'État, les membres du clergé devaient se soumettre aux « règlements de police qui, sans toucher à la foi et aux dogmes, importent à l'indépendance et à la dignité de la souveraineté temporelle " ${ }^{54}$. La majorité ne se rallia pas à ce point de vue. Le principe retenu fut celui de l'élection, afin de «mettre autant que possible en harmonie la constitution de l'Église et celle de l'État ${ }^{55}$; pour le représentant Cénac, l'élection était « une source vive » dans laquelle devaient venir «se retremper " toutes les institutions désireuses d'« obtenir force et respect aux yeux du peuple et $\left[d^{\prime}\right]$ accomplir la mission qui leur a été confiée par la Providence ${ }^{56}$. Tout en préconisant ce nouveau processus d'accès à l'épiscopat, le comité tenait absolument à éviter tout risque de schisme et entendait soumettre ses propositions à l'examen du Saint-Siège. Aux partisans du statu quo, il fut objecté que rien dans l'histoire ou dans les institutions de l'Église ne s'opposait au principe électif, comme le prouvaient la vie de l'Église primitive et l'élection du souverain pontife lui-même.

a déterminer le nombre et la qualité des électeurs - en veillant à ne pas renouveler les erreurs de la constitution civile du clergé - et le mode d'intervention de l'État; les évêques, estimait-on, exerçaient « une trop grande influence pour que l'État $p$ [ût] rester indifférent en pareille matière ${ }^{57}$. Diverses propositions furent examinées. Dans sa séance du 18 février 1849, le comité repoussa l'idée d'un corps électoral formé par des laïcs, s'agit-il de notables comme les maires, les fabriciens, les chefs de famille notoirement catholiques, et retint la solution suivante: dans chaque diocèse, les ecclésiastiques présenteraient trois candidats au chef de l'État, mais celui-ci pourrait faire porter son choix sur une autre personne.

Le comité s'intéressa aussi aux circonscriptions diocésaines. Un vœu porta sur la suppression de cinq archevêchés et la création de nouveaux évêchés, de manière à ce qu'il y eût désormais un évêché par département ${ }^{58}$. En ce qui concerne le nombre de succursales et de vicariats, le comité décida d'inviter le gouvernement à ne pas créer de nouvelles succursales pour des motifs politiques, mais à vérifier que les besoins invoqués pour l'érection de nouvelles paroisses étaient fondés; il n'entendait pas remettre en cause le principe de l'affectation de nouveaux vicaires à des paroisses surchargées. Il examina plus rapidement la situation du chapitre de la basilique de Saint-Denis ${ }^{59}$; en cas de suppression de ce chapitre, il souhaitait la fondation d'un nouveau chapitre de chanoines qui garantirait « la dignité du culte dans cette antique basilique » ${ }^{60}$.

Les membres du comité s'interrogèrent aussi sur le nombre des ordinations, qui dépassaient d'un tiers le nombre des ecclésiastiques décédés ${ }^{61}$. Certains voyaient un danger dans ce déséquilibre numérique; ils se demandaient si ces nouveaux prêtres n'étaient pas affectés à un ministère autre que le ministère paroissial et s'il était juste que tant de jeunes gens profitassent de l'exemption accordée aux ecclésiastiques par 
l'article 14 de la loi du 21 mars 1832. Aussi, ils souhaitaient une application stricte du vingt-sixième article organique, dont le second alinéa stipulait que "les évêques de France ne feront aucune ordination avant que le nombre des personnes à ordonner n'ait été soumis au gouvernement, et par lui agréé ${ }^{62}$. Ce à quoi il fut répondu que revenir à cette disposition serait " mettre la main à l'encensoir », que le clergé ne méritait pas tant de défiance et que plusieurs articles organiques n'avaient jamais été exécutés " parce que la pratique [avait] démontré le vide de leurs théories " ${ }^{63}$.

17 Le comité se montra attentif à la formation du clergé. Le fonctionnement des facultés de théologie existantes ne lui semblait pas satisfaisant; en outre, ces établissements n'étaient pas canoniques. Ses membres souhaitaient donc la fondation d'un haut enseignement théologique de qualité, grâce à la création de trois nouvelles facultés de théologie qui assureraient un " complément aux études des séminaires diocésains " ${ }^{64}$ et seraient établies à Paris, Lyon et Toulouse; ces innovations seraient introduites après concertation avec le Saint-Siège, afin de veiller à « l'orthodoxie de l'enseignement " ${ }^{65}$. Les professeurs de ces facultés, de même que les évêques devraient, à terme, tous être docteurs en théologie, tandis que les curés de première classe devraient être titulaires d'une licence; les études des séminaristes seraient sanctionnées par un examen de baccalauréat en théologie subi devant l'évêque ou son représentant. En ce qui concernait les facultés de théologie protestante, d'après le rapport de $\mathrm{M}$. Coquerel, celle de Strasbourg n'appelait pas de réformes. Les Églises réformées étaient favorables à un transfert de la faculté de théologie de Montauban à Paris ; le comité se montra favorable à ce souhait, mais jugea nécessaire d'attendre les vœux de l'assemblée des pasteurs réunis à Paris pour prendre une telle décision.

Le Comité des Cultes entendit un rapport que M. Grelier du Fougeroux présenta au nom de la sous-commission des pétitions, à propos de deux pétitions relatives à l'abolition du célibat des prêtres. Les pétitionnaires demandaient « un décret permettant aux prêtres de se marier sans recourir aux tribunaux $"{ }^{66}$. Quelques membres du Comité demandaient cette réforme avec acharnement, dit Pierre Pradié, qui semblait en concevoir un grand étonnement et partager la sévérité de M.Grelier du Fougeroux envers une telle revendication. À l'exigence du droit au mariage pour les prêtres - sur lequel le code civil est muet - on opposait les dispositions du sixième article organique, sanctionnant «l'attentat aux libertés, franchises et coutumes de l'Église gallicane», et du vingtsixième, interdisant l'ordination d'une personne ne possédant pas « les qualités requises par les canons reçus en France ${ }^{67}$. Or, le célibat fait partie de ces coutumes et canons. À dire vrai, dans ce domaine, la difficulté surgissait non pas lorsqu'un prêtre voulait se marier tout en poursuivant sa carrière sacerdotale - nous n'avons relevé aucun exemple de cette sorte -, mais quand, ayant abandonné le sacerdoce, il voulait prendre femme. Durant les premières années de la monarchie de Juillet, la chronique avait été nourrie par une affaire dont le souvenir plana longtemps sur la question du mariage des prêtres. En 1831, le tribunal de la Seine avait autorisé un abbé « défroqué ", l'abbé Dumonteil, à se marier civilement ; mais la Cour royale de Paris avait annulé cette décision le 14 janvier 1832 et l'ex-abbé Dumonteil, qui s'était pourvu en cassation avait été débouté le 24 février $1833{ }^{68}$. D'autres cas s'étaient encore présentés dans les années suivantes. Ainsi, en 1846, l'abbé Guicheteau, prêtre du diocèse de Luçon, avait-il abandonné le sacerdoce et avait-il souhaité se marier, mais le tribunal de Fontenay avait interdit à l'officier d'état civil de célébrer son union et l'ex-abbé Guicheteau était allé à Jersey, où le consul de France avait consenti à le marier ${ }^{69}$. Le Journal de la liberté religieuse signalait que quelques maires 
avaient accepté de marier des prêtres démissionnaires et que jamais les enfants nés de telles unions n'avaient été déclarés bâtards, ce qui prouvait la validité de l'union de leurs parents; cet argument fut repris par un membre du comité des Cultes, que Pierre Pradié ne nomme pas ${ }^{70}$.

Parmi les arguments invoqués pour s'opposer au mariage des prêtres, y compris de ceux qui avaient abandonné leur ministère, figurait la nature de l'ordination : obligé de tenir compte des canons de l'Église, l'État devait la considérer comme un sacrement indélébile, ineffaçable, résistant aux "caprices » et au "changement de volonté » ${ }^{71}$ de ceux qui l'avaient reçue. Un autre argument était avancé, la pratique de la confession auriculaire. "Quelle mère ne tremblerait pas, disait M. Grelier du Fougeroux, si celui qui a reçu les secrets de la conscience de sa fille pouvait, en sortant du saint tribunal, la conduire devant l'officier de l'état civil et devenir son époux! ${ }^{72}$. On se demandait aussi si, une fois marié, le prêtre renégat ne divulguerait pas des aveux destinés à demeurer enfouis : «Le peuple qui a cru aux serments du ministre de son culte et lui a livré les secrets de sa conscience a le droit de se plaindre, et l'État doit empêcher la violation d'un engagement sacré qui intéresse la moralité publique et la sécurité des familles " ${ }^{73}$. Par ailleurs, ajoutait M. Grelier du Fougeroux, la loi n'imposait pas le sacrifice «d'une partie des libertés naturelles " aux seuls ecclésiastiques; des laïcs aussi étaient concernés par de telles mesures, par exemple les marins et les soldats qui devaient rester célibataires durant toute la durée de leur service ; quant à l'interdiction de contracter une deuxième union tant que le premier conjoint était en vie, elle constituait bien aussi une mesure pesant sur la vie privée des individus. Le rapporteur était sans indulgence pour les pétitionnaires : «ils se sont trompés d'époque, écrivait-il ; ils n'ont pas compris que la révolution de 1848 ne s'est pas accomplie sous l'empire de l'esprit antireligieux qui dominait la génération qui nous a précédés. Ils ont oublié que la France ne veut plus de ces persécutions sournoises qui attaquent et cherchent à tuer le catholicisme sous prétexte de le protéger " ${ }^{74}$. D'après lui, d'ailleurs, le clergé aimait et respectait « la chaîne que ses faux amis voudraient briser ${ }^{75}$.

Un membre du comité proposa une solution intermédiaire entre le célibat obligatoire et la reconnaissance du droit au mariage. Il suggérait le vote d'une loi qui interdirait le mariage aux prêtres démissionnaires pendant les cinq années suivant leur renonciation au sacerdoce; au bout de cette période, ils seraient libres de se marier, à condition que ce ne fût pas avec une ancienne pénitente ${ }^{76}$. Mais, comme lui demanda l'un des ses collègues, " comment savoir de manière certaine si une femme a été une pénitente ou non? » La suggestion ne fut pas retenue.

21 La question qui retint le plus longuement et qui préoccupa le plus le comité des Cultes semble avoir été l'inamovibilité des desservants, objet de nombreuses pétitions, présentées par Pierre Pradié au comité des Cultes le 17 août 1848. Pradié n'était guère favorable aux pétitionnaires car, faisait-il remarquer, on ne possédait pas « d'exemple de prêtres dignes révoqués »; il ajoutait que si un prêtre ne convenait pas dans le poste où il avait été nommé, il était nécessaire de pouvoir le déplacer « pour cette raison bien simple que les prêtres sont faits pour les populations et non les populations pour les prêtres » ${ }^{77}$. La question est assez complexe et se rapporte à deux manières de considérer l'inamovibilité, l'une sous l'angle civil et l'autre sous l'angle canonique. D'après certains membres du comité, l'État ne pouvait accepter que les évêques eussent un droit exclusif de révocation ; une telle sanction, estimaient-ils, ne pouvait être prononcée avant qu'une officialité n'eût rendu un jugement pour des motifs d'ordre canonique. C'est pourquoi, 
d'après eux, les prélats auraient dû rétablir des tribunaux ecclésiastiques, comme Mgr Sibour l'avait fait lorsqu'il était évêque de Digne. D’ailleurs les pétitionnaires ne demandaient pas « une inamovibilité absolue, mais un jugement contradictoire " ${ }^{78}$ rendu par des officialités qui ne seraient toutefois pas les officialités d'Ancien Régime.

Le 11 novembre 1848, MM. Gavaret, Isambert et Mispoulet, membres du comité, ainsi que Pascal Duprat et Edgar Quinet, déposèrent à l'Assemblée nationale une proposition de loi visant à rendre aux desservants «les garanties dont ils jouissaient dans l'ancien droit public de la France». Pour les auteurs de ce texte, la République avait le devoir "de réintégrer dans leurs droits primitifs des pasteurs qui font jouir le plus immédiatement le peuple des bienfaits de la religion " ${ }^{79}$. Le 22 janvier 1849, le comité des Cultes examina la proposition et entendit ses auteurs. Isambert - qui n'était certainement pas un ami des prêtres - parla avec lyrisme de la vénération attachée à la personne du pasteur « qui a passé sa vie au sein d'une paroisse, qui a vu naître les enfants, marié les jeunes filles, donné la sépulture aux vieillards » et exprima sa défiance envers « les prêtres nomades » ${ }^{80}$, qu'il assimilait à des missionnaires. Tout en se prononçant pour "l'indépendance réciproque et absolue » de l'État et de l'Église, Pascal Duprat estima qu'en attendant que « le progrès des temps " amenât cette innovation, il fallait « rapprocher davantage notre société religieuse de notre société politique » et donner à l'Église une constitution voisine de celle de l'État en "ramen[ant] la démocratie dans l'Église " ${ }^{81}$. En outre, pour Pascal Duprat, élever le peuple exigeait d'élever les prêtres des campagnes, qu'il regardait comme « les magistrats de la conscience populaire " ${ }^{82}$. Si pour divers membres du comité, l'examen d'une telle question ne relevait pas de l'Assemblée nationale, la majorité estima que la situation du clergé « du second ordre » appelait « un sérieux examen et peut-être certaines réformes ${ }^{83}$.

Mgr Parisis et Mgr Fayet insistèrent pour un appel au Saint-Siège ; Pascal Duprat leur fit observer que l'Assemblée nationale était souveraine et n'avait « donc pas besoin de Rome pour abolir un décret de Bonaparte ", mais consentit à adopter cette solution ${ }^{84}$. La proposition de loi qu'il avait signée avec plusieurs de ses collègues fut reformulée ainsi : "L'article 31 de la loi organique du Concordat ${ }^{85}$ est rapporté en ce qui concerne la révocabilité des desservants. Il sera ouvert immédiatement, par le Gouvernement, des négociations avec le Souverain Pontife, pour régler les conditions d'après lesquelles seront rétablies l'inamovibilité des desservants et les officialités ecclésiastiques ${ }^{86}$. Lorsque Chapot présenta cette proposition à l'Assemblée le 26 mars 1849, il l'assortit de ce commentaire : «C'est bien le moins qu'on donne la parole à l'Église, qu'on la consulte et qu'on reconnaisse formellement que rien ne peut être tenté, en fait de changement de discipline, que par elle-même ou de son consentement " ${ }^{87}$.

Il fut donc demandé au comte de Falloux de bien vouloir se présenter devant le comité, car il semblait nécessaire de connaître l'opinion du ministre des Cultes avant de proposer à l'Assemblée l'ouverture de négociations avec Rome, qui ne pourraient aboutir qu'avec « le concours du pouvoir exécutif » ${ }^{88}$. Falloux vint devant le comité le 5 février 1849 ; il lui fit savoir que le gouvernement allait ouvrir des négociations avec le Saint-Siège et qu'il prendrait "pour point de départ les conclusions du comité des Cultes " ${ }^{89}$. Le 26 mars 1849 , Chapot présenta un rapport relatif à cette question à l'Assemblée nationale. Son texte reste empreint d'un très grand respect pour l'Église catholique et exprime une défiance certaine envers les partisans de l'inamovibilité des desservants. Un passage se rapporte aux prêtres qui, " éveillés par quelques publications d'une doctrine équivoque " se mirent à craindre pour leur indépendance et à croire leur ministère compromis, alors 
qu'aucune administration ne peut se montrer «plus paternelle » que celle des évêques ${ }^{90}$. Il demanda, au nom du comité, que les négociations avec Rome fussent «immédiates et promptement conduites»; ainsi la France prouverait-elle "à l'auguste pontife, si durement éprouvé, que l'antique esprit catholique de France survit à toutes les révolutions, et qu'elle n'a rien perdu de son respect et de son dévouement pour le SaintSiège $"{ }^{91}$. Puis ce dossier s'enlisa.

Inachevées et devenues rapidement caduques, les réformes introduites par la Seconde République n'en demeurent pas moins fondamentales. Un consensus se dégagea pour que la liberté de conscience cessât d'être un vain mot, pour que les fidèles des cultes minoritaires n'eussent plus à souffrir pour leur foi et ne se sentissent plus écrasés par le poids institutionnel de l'Église catholique, qui demeurait toutefois privilégiée en la personne de ses ministres.

Des représentants, qui n'étaient pas tous des amis de l'Église ni de la religion catholique, déployèrent de grands efforts pour faire pénétrer dans l'Église le vent de liberté qui soufflait à l'extérieur et pour lui insuffler un peu de l'esprit démocratique qui triomphait (temporairement) dans le siècle. La modération de nombreux laïcs et l'influence des ecclésiastiques, en particulier celle des évêques, empêcha le comité des Cultes et l'Assemblée nationale de tomber dans les mêmes erreurs que l'Assemblée nationale constituante de 1789-1791. Les constituants veillèrent à ne pas intervenir dans la vie ou dans la discipline de l'Église sans l'avis ou sans le consentement de celle-ci: ils craignaient bien trop de s'aliéner une partie du clergé et de renouveler l'expérience dramatique de la Grande Révolution.

Malgré le retrait de toute référence explicite à la religion catholique dans la Constitution - ce qui n'était pas anodin -, le respect envers Rome, l'Église et sa hiérarchie fut de mise durant les années 1848 et 1849. D'ailleurs, malgré la propagande activement menée durant la monarchie de Juillet, l'opinion éclairée, pas plus que l'opinion publique, n'était prête à des solutions nouvelles et radicales comme la séparation. En outre, les affaires religieuses de la nation n'étaient manifestement pas considérées comme une priorité, comme en témoigne la composition du comité des Cultes. Celui-ci compta assez peu de représentants de toute première importance; les grands noms semblent avoir été occupés ailleurs, dans d'autres comités, consacrés à des travaux qui correspondaient davantage aux préoccupations politiques et sociales immédiates, lesquelles, par la suite, tendirent à monopoliser l'attention des historiens.

\section{NOTES}

1.. Voir L'Ami de la religion, 24 février 1848, nº 4498, tome 136, pp. 497-498, cité par Paul CHRISTOPHE, L'Église de France dans la Révolution de 1848, Paris, les Éditions du Cerf, 1998, 
p. 19. Voir aussi Jean LEFLON, L'Église de France et la Révolution de 1848, Paris, Bloud et Gay, 1948, p. 15.

2. Pierre PRADIÉ, La question religieuse en 1682, 1790, 1802 et 1848 et historique complet des travaux du comité des Cultes de l'Assemblée constituante de 1848, Paris, Sagnier et Bray, 1849, p. 387.

3.. Voir Frank Paul BOWMAN, Le Christ romantique, Genève, Librairie Droz, 1973, 279 p., et Le Christ des barricades. 1789-1848, Paris, Les Éditions du Cerf, 1987, 361 p.

4.. Comte de FALLOUX, Mémoires d'un royaliste, tome I, La Restauration. La monarchie de Juillet. La Révolution de 1848, Paris, Librairie académique Perrin, 1925, pp. 254-255.

5.. Voir Joseph BRUGERETTE, Le prêtre français et la société contemporaine. Tome 1, La restauration catholique (1815-1871), Paris, P. Lethielleux, 1933, pp. 126-129.

6.. Actes officiels du Gouvernement provisoire dans leur ordre chronologique. Arrêtés, décrets, proclamations, etc., etc. Revue des faits les plus remarquables précédée du récit des événements qui se sont accomplis les 22, 23 et 24 février 1848, Paris, Barba-Garnot, 1848, p. 21.

7.. Circulaire du ministre de l'Instruction publique et des Cultes, 8 novembre 1848 ; ce document a été reproduit par L'Ère nouvelle, 11 novembre 1848 et est conservé dans divers dépôts d'archives départementales, par exemple, Archives départementales du Puy-deDôme, M 128. Voir Jacqueline LALOUETTE, «Célébrer la constitution de 1848 dans les départements de l'Allier, du Cantal, de la Haute-Loire et du Puy-de-Dôme », dans Jean-Éric IUNG [dir.], Fidélité républicaine et monde rural. 1848-1851, Actes du colloque d'Aurillac

(27-28 août 1999), Aurillac, Société des lettres, sciences et arts « La Haute-Auvergne », 2001, pp. 111-141.

8.. Voir Cinquante ans de recherches sur 1848, Francis DEMIER et Jean-Luc MAYAUD [dir.], Revue d'Histoire du XIX ${ }^{e}$ siècle, n 14, 1997, pp. 129-199.

9.. Le Semeur était considéré comme un journal protestant ; ainsi La Démocratie pacifique du 11 novembre 1843 le présentait-elle comme « l'un des organes du protestantisme en France ». Cependant Le Semeur lui-même refusait de se voir ainsi défini et disait être « purement et simplement un journal chrétien », Le Semeur, 11 décembre 1833.

10. Voir Essai sur la manifestation des convictions religieuses et sur la séparation de l'Église et de l'État envisagée comme conséquence nécessaire et comme garantie du principe, Paris, Paulin, 1842.

11.. Voir L'Ami de la religion, $\mathrm{n}^{\circ}$ 1931, 12 avril 1832, p. 491 ; et Pierre-François AUDRY DE PUYRAVEAU, Opinion prononcée par M. Audry de Puyraveau sur le budget des finances le 26 janvier 1833, Paris, Imprimerie d'Auguste Mie, 1833.

12. Voir la lettre adressée par Isambert au Journal de la liberté religieuse, $n^{\circ} 9-10$, janvierfévrier 1844, p. 649 : « Je suis le premier qui ait osé parler à la tribune nationale de la séparation de l'Église et de l'État ».

13. Séance du 3 mai 1845, voir Alphonse de LAMARTINE, La France parlementaire (1834-1851). Euvres oratoires et écrits politiques par Alphonse de Lamartine, précédées d'une étude sur la vie et les œuvres de Lamartine par Louis Ulbach, Paris, Librairie internationale A. Lacroix, Verboeckhoven et Compagnie, 1864-1865, 6 volumes, tome 4, pp. 158-171.

14.. Charles-Antoine TESTE, Projet de constitution républicaine et déclaration des principes fondamentaux de la société précédés d'un exposé des motifs, Bruxelles, 1836, art. 180, p. 80.

15.. Charles-François CHEVÉ, Programme démocratique, ou résumé d'une organisation complète de la démocratie radicale, Imprimerie de A. Appert, 1840, p. 39. 
16.. Art. 6 de la Charte du 4 juin 1814 : «Cependant la religion catholique, apostolique et romaine est la religion de l'État ".

17.. Art. 5 de la Charte du 14 août 1830 : « Chacun professe sa religion avec une égale liberté, et obtient pour son culte la même protection »; art. 6 : « Les ministres de la religion catholique, apostolique et romaine, professée par la majorité des Français, et ceux des autres cultes chrétiens, reçoivent des traitements du Trésor public ». Les rabbins, eux, ne reçurent un traitement qu'à partir de 1831.

18.. Le Semeur, 16 janvier 1833.

19.. Le Semeur, 14 septembre 1836.

20.. Voir Liberté des cultes. Affaire des protestants de Senneville, Paris, Imprimerie de F. Locquin, 1843, Bib. nat., 4 Ld $^{176} 840$. Le pasteur Roussel s'installa ultérieurement à Mansle (Charente) et fut de nouveau poursuivi pour avoir formé une association religieuse sans l'autorisation du Gouvernement. Il fut condamné par le tribunal de Ruffec, mais la Cour d'Angoulême lui fut favorable en appel, Le Semeur, 13 janvier 1847, p. 15 et 3 mars 1847, p. 66. Pour ce pasteur à l'étonnante personnalité, voir André ENCREVÉ, Les protestants en France de 1800 à nos jours. Histoire d'une réintégration, Paris, Éditions Stock, pp. 78-79.

21.. Si, même en région d'habitat dispersé, les catholiques étaient toujours assurés de trouver un édifice cultuel en un lieu proche, ou relativement proche, de leur domicile, il n'en allait pas de même pour les protestants de l'Église luthérienne ou de l'Église réformée, toutes deux reconnues. A fortiori, dans les zones de dissémination, les fidèles protestants pouvaient se trouver très éloignés de tout temple, d'où les initiatives prises pour célébrer des offices sans tenir compte des règles établies.

22. Voir Le Semeur, 17 février 1847, p. 56 et 31 mars 1847, p. 103.

23.. Actes officiels du Gouvernement provisoire..., ouv. cité, pp. 69-70.

24.. Le préambule est précédé des quelques lignes suivantes : «Au nom du peuple français, l'Assemblée nationale a adopté... » ; la référence à Dieu ne porte donc pas sur l'ensemble de la Constitution, mais sur les grands principes énoncés dans le préambule. Pour la valeur de ce préambule, voir Patrice ROLLAND, « De l'art du préambule », dans Jean BART, Jean-Jacques CLERE, Claude COURVOISIER et Michel VERPEAUX [dir.], La Constitution du 4 novembre 1848 : l'ambition d'une République démocratique. Actes du colloque de Dijon, 10 et 11 décembre 1998, Dijon, Éditions universitaires de Dijon, 2000, pp. 143-186. 25.. Mgr Fayet (1786-1849) était évêque du diocèse d'Orléans depuis 1843 ; il fut élu représentant de la Lozère en avril 1848, voir L'épiscopat français depuis le concordat jusqu'à la séparation (1802-1905), ouvrage publié sous la direction de la société bibliographique avec le concours de 90 collaborateurs diocésains, préface de Mgr Baunard, Paris, Librairie des SaintsPères, 1907, pp. 431-432.

26. Aucun parlementaire du nom de ce nom ne figure dans Adophe ROBERT, Edgar BOURLOTON et Gaston COUGNY [dir.], Dictionnaire des parlementaires français comprenant tous les membres des Assemblées françaises et tous les ministres français depuis le $1^{\mathrm{er}}$ mai 1789 jusqu'au $1^{\mathrm{er}}$ mai 1889, Paris, Bourloton éditeur, 1891, 6 volumes.

27. Célèbre pour son ouvrage intitulé Terre et ciel, Jean Reynaud (1806-1863) appartenait à la Gauche modérée ; il fut élu en mars 1848 dans le département de la Moselle ; voir Adophe ROBERT, Edgar BOURLOTON et Gaston COUGNY [dir.], Dictionnaire des parlementaires français..., ouv. cité, volume 5, p. 130.

28. La Constitution de 1848 précédée $1^{\circ}$ du rapport fait au nom de la commission par le citoyen A. Marrast $2^{\circ}$ du projet de constitution, avec les divers amendemens proposés, soit qu'ils aient été adoptés ou rejetés, et les noms des orateurs entendus dans la 
discussion de chaque article $3^{\circ}$ de la révision $4^{\circ}$ du résultat des scrutins qui ont été demandés sur les questions les plus importantes, suivie du décret relatif à l'élection du Président de la République, Paris, chez Édouard Proux et Compagnie, novembre 1849, pp. 26-29.

29.. Pierre Leroux était un esprit profondément religieux ; il estimait qu'une religion était indispensable à la vie d'un peuple et jugeait absurde le principe de la séparation entre le spirituel et le temporel. Mais il souhaitait un culte national capable d'assurer l'unité de la société tout en laissant l'individu libre et, pour lui, le christianisme ne pouvait absolument pas remplir cette mission. Voir Pierre LEROUX, D'une religion nationale ou du culte, Boussac, Imprimerie de Pierre Leroux, 1846.

30.. Jean Lavallée (1806-1879), ancien membre de la société « Aide-toi le ciel t’aidera », représentant de la Charente, sous-commissaire du Gouvernement provisoire à Ruffec ; voir Adophe ROBERT, Edgar BOURLOTON et Gaston COUGNY [dir.], Dictionnaire des parlementaires français..., ouv. cité, volume 3, pp. 639-640. De son côté, Lamennais publia un projet de constitution dont l'article 14 rendait tous les cultes indépendants de l'État ; celui-ci ne devait en salarier aucun, mais avait le devoir de les protéger tous, voir La Vraie République, 7 mai 1848.

31.. En 1845, le chevalier Massias avait adressé à la Chambre des députés une pétition qui visait certainement à la reconnaissance de nouveaux cultes. Elle était ainsi libellée : «J'ai l'honneur de vous prier de vouloir bien prendre l'initiative d'une loi qui détermine le nombre de citoyens dont l'adhésion aux mêmes croyances religieuses est nécessaire pour constituer une religion reconnue par l'État et pour donner à leur culte droit à la protection dont les autres jouissent "; Chevalier MASSIAS, Remède catholique et constitutionnel à l'ultramontanisme, Paris, Abel Ledoux, 1845, p. 3, Bib. nat., Ld4 5025. 32.. La Constitution de 1848 précédée..., ouv. cité, p. 42. Bouzique (1801-1877) était un démocrate modéré ; il fut élu représentant du Cher en mars 1848 : il fut maire de Bourges en 1848, voir Adophe ROBERT, Edgar BOURLOTON et Gaston COUGNY [dir.], Dictionnaire des parlementaires français..., ouv. cité, volume 1, pp. 461-462.

33.. Idem, volume 1, p. 451.

34.. Alexandre-François-Auguste Vivien (1799-1854). Député de 1833 à 1848, il appartenait au courant de l'opposition dynastique. Élu représentant de l'Aisne en 1848. ministre dans le gouvernement Cavaignac, il se démit de toutes ses fonctions en 1851 ; idem, volume 5 , pp. 543-544.

35.. Pierre PRADIÉ, La question religieuse..., ouv. cité, p. 181.

36.. Parallèlement aux bureaux traditionnels, l'Assemblée « se groupa en quinze comités spéciaux intitulés : comité des finances, comité des affaires étrangères, comité de l'Instruction publique, comité du travail, etc. Chaque représentant s'inscrivait à l'un de ces comités, selon ses études et sa préférence ». Comte de FALLOUX, Mémoires d'un royaliste, ouv. cité, tome 1, pp. 292-293.

37.. Dont la liste se trouve dans Pierre PRADIÉ, La question religieuse..., ouv. cité, p. 44.

38.. Mgr Parisis (1795-1866) fut évêque de Langres de 1835 à 1851, puis évêque d'Arras de 1851 à 1866 ; il fut élu représentant par le département du Morbihan en 1848. Mgr Graveran (1793-1855) avait été nommé évêque de Quimper en 1840 et élu représentant dans le Finistère en 1848, voir L'épiscopat français..., ouv. cité, pp. 282-283 et pp. 493-494. Ces trois évêques étaient surnommés respectivement Magnificat, Aedificat et Laetificat, Comte de FALLOUX, Mémoires d'un royaliste, ouv. cité, p. 285. D'après Louis Veuillot, les trois prélats furent « utiles au comité des Cultes, agité d'un esprit d'innovation que leur prudence a pu conjurer », et leur présence exerça un effet 
bénéfique sur l'Assemblée, rendant « les orateurs plus réservés et l'Assemblée plus bienveillante », Louis VEUILLOT, Mgr Parisis, Paris, E. de Soye, 1864, p. 11.

39.. D'après le $\mathrm{n}^{\circ} 1$ du Vrai Catholique (novembre 1848), outre les trois évêques, le comité des Cultes comptait cinq prêtres, et non pas trois, p. 3.

40.. Voir Jean SIGNOREL, Portraits politiques. Arnaud de l'Ariège, reprint Lacour-Rediviva, Nîmes, Lacour, 2001, 24 p. Arnaud de l'Ariège, dit l'auteur (p. 23), était tenu en suspicion par les républicains anticléricaux à cause de son catholicisme et par les catholiques à cause de son républicanisme.

41.. Le Vrai catholique, $\mathrm{n}^{\circ} 1$, novembre 1848 , p. 3.

42.. Pierre Pradié (1816-1892) ; élu représentant dans l'Aveyron en 1848, il appartenait à l'école buchézienne, voir Adophe ROBERT, Edgar BOURLOTON et Gaston COUGNY [dir.], Dictionnaire des parlementaires français..., ouv. cité, volume 5, pp. 39-40.

43.. Pierre PRADIÉ, La question religieuse..., ouv. cité, p. 443.

44.. Pierre Pradié ayant parfois désigné les orateurs par la lettre « $M$ » - soit qu'il n'ait pas voulu les nommer, soit qu'il ne se soit plus souvenu qui avait pris la parole en telle ou telle occurrence -, il nous est impossible de savoir qui étaient les partisans de ces solutions radicales. On peut supposer qu'Isambert ne fut pas le dernier à parler en ce sens.

45.. Pierre PRADIÉ, La question religieuse..., ouv. cité, p. 37.

46. Idem, p. 31.

47. Idem, p. 33.

48. Ibidem.

49. Idem, p. 438.

50. Ibidem.

51. Idem, p. 187.

52.. Idem, p. 190.

53.. Pour l'essentiel, nous rendons compte des discussions du comité des Cultes dans l'ordre adopté par Pierre Pradié ; toutefois nous avons opéré certains regroupements thématiques, notamment pour présenter les questions financières. Nous empruntons nos citations tantôt au détail des discussions, tantôt au résumé que l'auteur a présenté à la fin de son ouvrage.

54.. Pierre PRADIÉ, La question religieuse..., ouv. cité, p. 144.

55. Idem, p. 145.

56. Idem, p. 165.

57. Idem, p. 147.

58.. Si le principe d'un évêché par département était globalement respecté, il y avait quelques exceptions, qui existent d'ailleurs toujours ; par exemple, le département des Deux-Sèvres ne correspond pas à un évêché et son territoire dépend du diocèse de Poitiers.

59.. Ce chapitre avait été érigé par un décret du 20 février 1806 ; il comprenait des évêques de plus de soixante ans qui ne pouvaient plus assumer leurs fonctions et dont le nombre, primitivement fixé à dix, avait ensuite été porté à douze. La Restauration y adjoignit des chanoines et ce chapitre prit le nom de Chapitre royal de Saint-Denis, voir Chanoine de ROQUETAILLADE, Saint-Denis, Paris, Letouzey et Ané, 1924, pp. 66, 130-131. 60.. Pierre PRADIÉ, La question religieuse..., ouv. cité, p. 441.

61.. Cette indication est donnée pour les années 1827-1847. Pendant cette période, 20221 prêtres étaient décédés et 33571 avaient été ordonnés, Pierre PRADIÉ, La question religieuse..., ouv. cité, p. 217. 
62.. Bernard ARDURA, Gérard CHOLVY et Cardinal BILLÉ, Le concordat entre Pie VII et Bonaparte, Paris, Éditions du Cerf, 2001, p. 81.

63.. Pierre PRADIÉ, La question religieuse..., ouv. cité, p. 218.

64.. Idem, p. 212.

65.. Ibidem.

66.. Assemblée nationale constituante. Session de 1849. Rapport fait par M. Grelier du Fougeroux au comité des Cultes au nom de la sous-commission des pétitions sur deux pétitions relatives au célibat ecclésiastique, Nantes, Imprimerie Vincent Forest et Émile Grimaud, 1864, p. 8.

67.. Vers 1813, Napoléon aurait eu l'intention, dit Pierre Pradié, de demander au Conseil d'État une sanction pénale contre les prêtres qui se marieraient et une loi déclarant bigames les prêtres mariés. Le Conseil d'État y était favorable, mais l'effondrement de l'Empire survint avant qu'il n'eut rédigé ces textes, Pierre PRADIÉ, La question religieuse..., ouv. cité, p. 258.

68. Assemblée nationale constituante. Session de 1849. Rapport fait par M. Grelier du Fougeroux..., ouv. cité, p. 8.

69.. Journal de la liberté religieuse, mai 1843.

70.. Ibidem et Pierre PRADIÉ, La question religieuse..., ouv. cité, p. 263.

71.. Ibidem.

72.. Assemblée nationale constituante. Session de 1849. Rapport fait par M. Grelier du Fougeroux..., ouv. cité, p. 9.

73. Ibidem.

74. Idem, pp. 8-9.

75. Idem, p. 9.

76.. Pierre PRADIÉ, La question religieuse..., ouv. cité, p. 263.

77. Idem, pp. 343-344.

78. Idem, p. 350.

79. Idem,. p. 359.

80. Idem, p. 360.

81. Idem, p. 365.

82. Idem, p. 366.

83.. Idem, p. 384.

84.. Idem, p. 367.

85.. Le deuxième alinéa du 31ème article organique était ainsi libellé : « Ils [les vicaires et desservants] seront approuvés par l'évêque et révocables par lui », voir Bernard ARDURA, Gérard CHOLVY et Cardinal BILLÉ, Le concordat entre Pie VII et Bonaparte, ouv. cité, p. 81.

86.. Pierre PRADIÉ, La question religieuse..., ouv. cité, p. 385. Il est assez piquant de constater que le Saint-Siège aurait pu être consulté pour la modification de l'un des articles organiques, alors qu'il n'avait jamais admis la validité de ces derniers, que Bonaparte avait fait rajouter unilatéralement et autoritairement à la convention signée avec Pie VII, le 26 messidor an IX.

87. Ibidem.

88. Idem, p. 372.

89. Ibidem.

90. Ibidem, p. 381.

91.. Ibidem, p. 386. Le compte rendu de Pierre Pradié se clôt sur ces informations. Dans l'état actuel de notre documentation, nous ne savons pas ce qu'il advint des travaux et des 
conclusions adoptées par le comité des Cultes. Une recherche complémentaire s'impose aux Archives nationales et, peut-être, aux Archives du Vatican.

\section{RÉSUMÉS}

Quelques épisodes religieux de la Révolution de 1848 sont bien connus, par exemple le transfert du Christ des Tuileries à l'église Saint Roch ou la bénédiction des arbres de la Liberté. Mais, d'une manière générale, les historiens de 1848 ont prêté une plus grande attention à la religiosité quarante-huitarde ou à l'histoire politique, économique et sociale de la Seconde République qu'à ses aspects religieux institutionnels. Ceux-ci ont cependant été importants. Dès le mois de mars, l'Assemblée constituante a assuré le triomphe de la liberté de conscience en levant les sanctions prononcées antérieurement à l'encontre de fidèles de cultes non concordataires. La constitution du 4 novembre 1848, promulguée "en présence de Dieu ", conserve les cultes concordataires, mais supprime toute référence propre à la religion catholique. Innovation importante, son article 7 envisage que de nouveaux cultes puissent être reconnus à l'avenir. Enfin, au sein de l'Assemblée constituante, se met en place un Comité des cultes, qui comprend, entre autres, trois évêques, trois abbés, quelques représentants du catholicisme intransigeant et du catholicisme libéral, quelques anticléricaux, un protestant. Ce Comité réfléchit à une possible refonte du concordat, en s'attachant particulièrement à quelques points : le budget des cultes, le célibat des prêtres, les procédures disciplinaires au sein de l'Église, la modification du statut des desservants, que certains membres voudraient rendre inamovibles. Démocratiser l'Église - avec l'aval de Rome -, afin d'adapter ses structures à celles de la jeune République, tel est le vœu essentiel d'une partie du Comité, dont les travaux, finalement, ne porteront aucun fruit.

The religious policy of the Second Republic. Some religious events of the 1848 Revolution are well-known to historians, for example the transfer of the "Christ of the Tuileries" to the Church of Saint-Roch, or the blessing of the liberty trees. But generally the historians of the 1848 Revolution have either paid attention to the religiosity of the '48ers or to the political, economic and social history of the Second Republic, rather than to issues of religious institutions. However, these are quite important. As early as March the Provisional Government guaranteed the triumph of religious freedom, by lifting the sanctions, which had previously been imposed against members of faiths not included in the Concordat. Promulgated "in the presence of God," the Constitution of 4 November 1848 maintained the legal existence of the faiths of the Concordat, but all special references to Catholicism were suppressed; an important innovation - Article 7 of the Constitution - envisaged that new faiths might be included in the Concordat in the future. The Committee on Religions was at the heart of the Constituant Assembly; among others, it included three bishops, three vicars, some representatives of liberal Catholicism, as well as of intransigent Catholicism, a few anticlerical members, and a Protestant. This committee considered modifying the Concordat, focusing on a few key issues: the religious budget, the celibacy of priests, the statute of parish priests - whom certain members of the Committee would have liked to make immovable -, the disciplinary procedures at the heart of the church. part of the Committee wanted to democratize the Church - with the approval of Rome adapting its structures to those of the young Republic. The work of the Committee on Religions, which was suspended in May 1849, never found any resonance and never had any consequences. 


\section{AUTEUR}

JACQUELINE LALOUETTE

professeur à l'Université Paris 13 